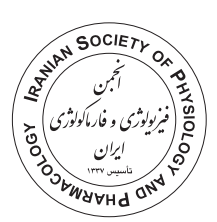

\title{
High- and moderate-intensity training are equipotent in abrogating myocardial inflammation in rats fed a Western diet
}

\author{
Abdulbaset Maroofi, Arsalan Damirchi* (iD
}

Department of Exercise Physiology, Faculty of Physical Education \& Sport Sciences, University of Guilan, Rasht, Iran

\begin{abstract}
Introduction: Western diet (WD) activates inflammatory pathways in the myocardium, where jeopardizes contractile function. This study aimed to compare the anti-inflammatory effects of high-intensity interval training (HIIT) and moderate-intensity continuous training (MICT) in rats fed a WD.

Methods: Wistar rats were assigned to the six groups: normal diet (ND)+HIIT, WD+HIIT, ND+MICT, WD+MICT, sedentary fed a ND or WD (SED+ND and SED+WD, respectively). HIIT and MICT were performed on a motorized treadmill, five consecutive days/week for 12 weeks. In these animals, visceral fat mass and myocardial expression of pro-inflammatory cytokines i.e. tumor necrosis factor-alpha (TNF- $\alpha$ ) and myeloperoxidase (MPO) were measured. Western blotting was performed to identify cardiac protein expression.

Results: WD+SED significantly increased visceral fat mass compared with ND+SED. WD+SED significantly resulted in TNF- $\alpha$ over-expression compared with ND+SED. There were no significant differences in MPO expression between WD+SED and ND+SED. In trained groups, visceral fat mass and TNF- $\alpha$ expression were lower in WD+HIIT and WD+MICT compared with WD+SED, with similar effects between HIIT and MICT modes. MPO expression was significantly lower in ND+HIIT and ND+MICT compared with ND+SED, with similar effects between HIIT and MICT modes.

Conclusion: WD co-existing with SED paves the way to a pro-inflammatory milieu in the heart. HIIT and MICT exert similar anti-inflammatory effects on the myocardium; therefore, aerobic training, regardless of modality or intensity, can be a practical solution for those who regularly consume WD.
\end{abstract}

\section{Introduction}

Cardiovascular disease (CVD) is a grave health concern that jeopardizes the everyday activities and life expectancy of approximately one-third of the world pop-

\author{
Keywords: \\ Cardiac inflammation \\ Exercise training \\ Obesity
}

Western diet

\footnotetext{
* Corresponding author: Arsalan Damirchi, damirchi@guilan.ac.ir

Received 31 December 2020; Revised from 22 January 2020; Accepted 1 February 2020
}

Citation: Maroofi A, Damirchi A. High- and moderate-intensity training are equipotent in abrogating myocardial inflammation in rats fed a Western diet. Physiology and Pharmacology 2021; 25: 306-313. http://dx.doi.org/10.52547/ppj.25.4.1 
crose (Western-style diet, WD) or high-fat diet (HFD) are well-recognized risk factors for metabolic disturbances and CVD (Carbone et al., 2015; McGavock et al., 2006; Oikonomou et al., 2018; Panagiotakos et al., 2009). Excessive WD or HFD consumption accelerates overall or central obesity, particularly with a co-existing sedentary lifestyle (Costa et al., 2011; Moreno-Fernández et al., 2018). Detrimental effects of abdominal (visceral) obesity are accompanied by accentuating systemic inflammation (Fontana et al., 2007; Lumeng and Saltiel, 2011). In fact, visceral fat can activate a network of inflammatory pathways (Gleeson et al., 2011). Chronic inflammation contributes to the onset of CVD (Pedersen, 2017).

Inflammation can lead to the enhancement of pro-inflammatory cytokine levels. Studies have shown that WD or HFD can trigger the expression of tumor necrosis factor-alpha (TNF- $\alpha$ ) in rodent myocardium (Chen et al., 2020; Kesherwani et al., 2015). Over-expression of TNF- $\alpha$ has been shown to impair myocardial contractile function (Meldrum, 1998). In recent years, myeloperoxidase (MPO) has emerged as a significant mediator of CVD (Koeth et al., 2013). MPO, a member of the heme protein superfamily, synthesized by leukocytes and released from azurophilic granules during inflammatory processes, mainly due to leukocyte activation (Kalász et al., 2015). The pro-inflammatory cytokine MPO inhibition reduces ventricular dysfunction in rats with myocardial infarction (Ali et al., 2016). There are only a few studies that have described the relationship of MPO with cardiovascular abnormalities in the context of HFD. In a study, Santana et al. (2014) showed that HFD-induced aortic stiffness is associated with an increase in MPO activity. However, the actual impact of WD on cardiac MPO expression is unknown.

Exercise training plays a fundamental role in maintaining cardiovascular health, particularly in the presence of medical conditions, such as obesity and diabetes (Mishra et al., 2011; Nystoriak and Bhatnagar, 2018). In fact, exercise training not only sustains optimal myocardial performance by fostering beneficial adaptations (Ellison et al., 2012; Shephard and Balady, 1999) but also confers protective effects against cardiac dysfunction imposed by myocardial infarction (Xi et al., 2016) or metabolic disturbances (Mostarda et al., 2012). It has been demonstrated that exercise training can attenuate cardiac abnormalities in animals fed a WD or HFD
(Chen et al., 2019; Kesherwani et al., 2015). It is speculated that exercise training limits HFD or WD-imparted cardiac disorders, in part, by repressing pro-inflammatory cytokines in rodent hearts, given the decisive role of inflammation in the pathogenesis of myocardial dysfunction (Carbone et al., 2015). This concept is supported by animal research. In a study, Kesherwani et al. (2015) found that aerobic training reduces cardiac TNF- $\alpha$ over-expression linked to HFD, but their assessments were limited to swimming exercise. Still, more studies are essential to establish an optimal training modality or intensity to enhance the effectiveness of exercise training in abrogating WD-induced cardiac inflammation. Accordingly, diversified aerobic training approaches, such as high-intensity interval training and moderate-intensity continuous training (HIIT and MICT, respectively), need to be compared. HIIT and MICT are often proving useful in cardioprotection (Hafstad et al., 2013; Verboven et al., 2019); however, may exhibit different anti-inflammatory effects in the context of WD. To the best of our knowledge, no study has reported the impact of HIIT vs. MICT on cardiac TNF- $\alpha$ and MPO expression in rats fed a WD.

Therefore, this study aimed to compare the effects of HIIT and MICT performed for 12 weeks on visceral fat mass and cardiac TNF- $\alpha$ and MPO protein expression in rats fed a WD or normal diet (ND).

\section{Materials and methods}

\section{Ethical approval}

The principles for animal research adopted by the American Physiological Society were rigidly followed (American Physiological Society, 2002), and all experimental procedures were approved by the National Research Ethics Committee of Guilan University of Medical Science. Approval ID: IR.GUMS.REC.1397.264

\section{Experimental design and sampling}

Sixty male Wistar rats (7-8 weeks old; initial body weight 250g) were supplied from Razi Vaccine and Serum Research Institute (Iran). After acclimatization for a 1-week, animals were randomly divided into the following six groups, each containing 10 rats: ND+HIIT, WD+HIIT, ND+MICT, WD+MICT, sedentary fed a ND or WD (SED+ND and SED+WD, respectively). WD (TD.88137-Envigo) composed of 42\% fat, $42.7 \%$ sucrose and $0.2 \%$ cholesterol, whereas ND 
(TD.08485-Envigo) contained 13\% fat and 67.9\% carbohydrate. All animals followed their exercise training or SED schedules for 12 weeks in a controlled environment with free access to ND or WD and freshwater. At the end of the experimental period, the rats were sacrificed under anesthesia induced by a mixture of ketamine and xylazine. Left ventricular tissue was snap-frozen and stored at $-80^{\circ} \mathrm{C}$ until Western blotting. Visceral fat, including mesenteric, epididymal and bilateral retroperitoneal were carefully separated from the abdominal cavity (Catta-Preta et al., 2012). All the purified fats were weighed together, using an Analytical Balance with 0.1 mg resolution (A\&D, Japan).

\section{Exercise intervention}

HIIT and MICT were performed on a motorized treadmill ( $15^{\circ}$ inclination), five consecutive days/week for 12 weeks as described earlier by Hafstad et al. (2013). Before engaging rats in the exercise of HIIT or MICT, and at the start of weeks of 4 and 8 training program, maximal (\%100) physical capacity (MPC) of animals was estimated according to previous studies (Machado et al., 2017). MPC was used to adjust the intensity of exercise bouts throughout the study. HIIT consisted of ten bouts of $4 \mathrm{~min}$ intense running (85-90\% MPC), interspersed by $2 \mathrm{~min}$ jogging ( $50 \% \mathrm{MPC}$ ). MICT consisted of an endurance running at $65-70 \%$ of MPC. Total running time in HIIT sessions was $60 \mathrm{~min}$, whereas in MICT protocol reached close to $100 \mathrm{~min}$. HIIT and MICT regimens were matched by volume. In fact, the total covered distances each session were similar in HIIT and MICT (Delwing-de Lima et al., 2018).

\section{Western blot analysis}

Western blotting was performed as described previ- ously (Chavali et al., 2014; Kesherwani et al., 2015). In brief, left ventricular tissue samples were homogenized in an ice-cold RIPA lysis buffer $(50 \mathrm{mM}$ Tris- $\mathrm{HCl}[\mathrm{pH}$ 7.5], $1 \%$ SDS, $150 \mathrm{mM} \mathrm{NaCl}, 0.5 \%$ sodium deoxycholate, $1 \%$ NP-40) supplemented with protease inhibitor cocktail (Sigma Aldrich). Using Bradford assay, the concentration of total protein was determined. Typically, $20 \mu \mathrm{g}$ of total protein were separated and resolved using polyacrylamide gel electrophoresis, then transferred to polyvinylidene difluoride membranes. Subsequently, the membranes were incubated with primary antibodies (Santa Cruz Biotechnology, USA) against anti-TNF- $\alpha$ (Cat. \#: sc-130349) and anti-MPO (Cat. \#: sc-390109) at a 1:1000 dilution followed by Goat anti-Rabbit HRP secondary antibody (Santa Cruz Biotechnology, USA) at a 1:5000 dilution. Western blot bands were visualized using the chemiluminescent reagents (Amersham Biosciences, USA). Bands intensity was quantified using ImageJ software (NIH, version 1.62). Cardiac $\beta$-actin expression was used to normalize the results.

\section{Statistical analysis}

All statistical analyses were performed using SPSS. The Shapiro-Wilk test was used to assess the normality of data. Experimental groups were compared using one-way ANOVA with Tukey's multiple post-hoc test. Statistically significant differences were set at $P<0.05$. All values are presented as mean \pm SEM.

\section{Results}

HIIT and MICT are similarly effective in obviating WD-induced visceral fat mass accumulation

As shown in Figure 1, compared with ND+SED, WD+SED exhibited a significant increased visceral fat mass $(P<0.05)$. Visceral fat mass was significant-

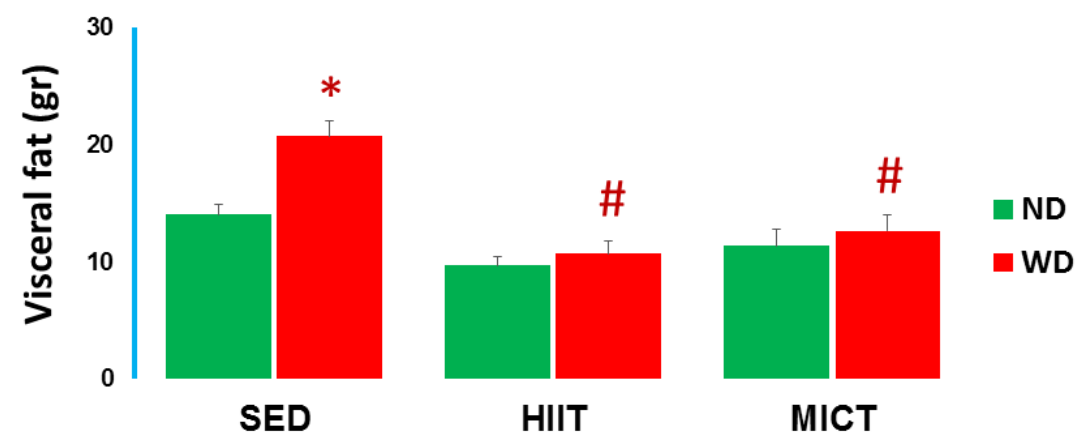

FIGURE 1. Visceral fat mass after high-intensity interval training (HIIT) and moderate-intensity continuous training (MICT) in rats fed a Western (WD) or normal (ND) diet. Values are expressed as mean \pm SEM. ${ }^{*} P<0.05$ vs. ND+SED; ${ }^{\sharp} P<0.05$ vs. WD+SED. SED: sedentary. 

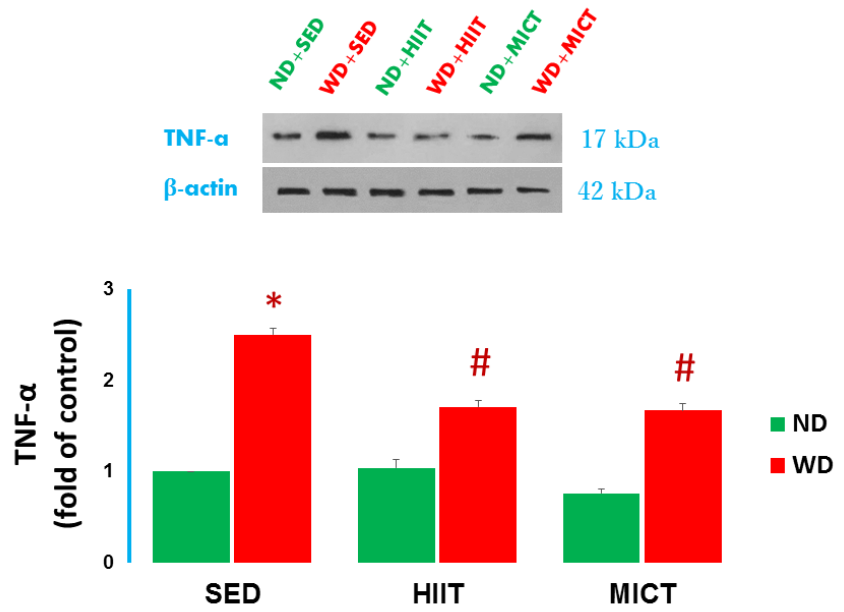

FIGURE 2. Cardiac tumor necrosis factor-alpha (TNF- $\alpha$ ) protein expression after high-intensity interval training (HIIT) and moderate-intensity continuous training (MICT) in rats fed a Western (WD) or normal (ND) diet. Values are expressed as mean \pm SEM. ${ }^{*} P<0.05$ vs. ND+SED; ${ }^{\sharp} P<0.05$ vs. WD+SED. SED: sedentary.

ly lower in WD+HIIT and WD+MICT compared with WD+SED $(P<0.05)$, with no significant differences between training modalities. HIIT and MICT caused no significant changes in visceral fat mass of the ND rats. When final total body mass (data not shown) was compared between ND and WD, no significant differences were observed. Final total body mass was significantly lower in WD+HIIT compared with WD+SED $(P<0.05)$.

Either HIIT or MICT is incisive enough to ameliorate WD-induced cardiac TNF- $\alpha$ over-expression

As shown in Figure 2, compared with ND+SED, WD+SED exhibited a significant increased TNF- $\alpha$ expression $(P<0.05)$. TNF- $\alpha$ expression was significantly lower in WD+HIIT and WD+MICT compared with WD+SED $(P<0.05)$, with no significant differences between training modalities. HIIT and MICT caused no significant changes in TNF- $\alpha$ expression of the ND rats.

HIIT and MICT similarly modifies cardiac MPO expression in rats fed a $N D$

As shown in Figure 3, no differences in MPO expression were observed between WD+SED and ND+SED. MPO was significantly lower in ND+HIT and ND+MICT compared with ND+SED $(P<0.05)$, with no significant differences between training modalities. HIIT and MICT caused no significant changes in MPO expression of the WD rats.
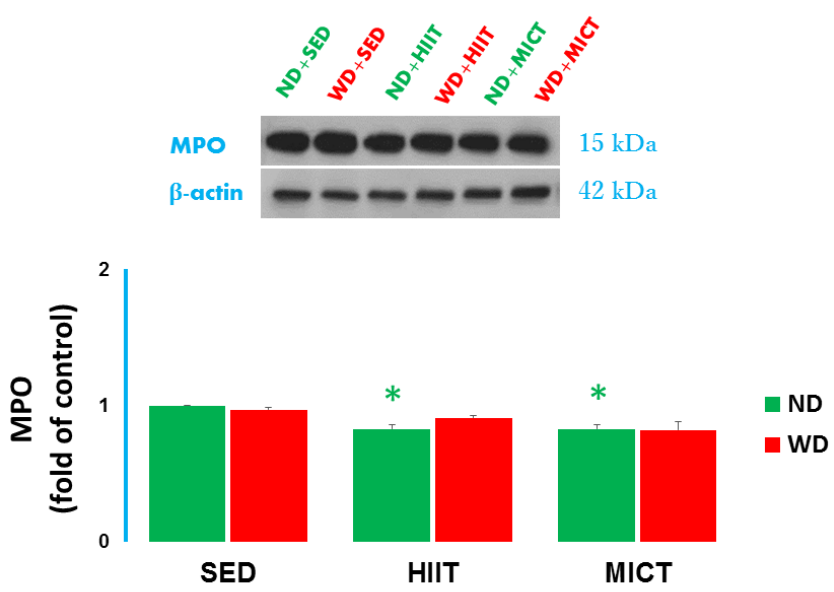

FIGURE 3. Cardiac myeloperoxidase (MPO) protein expression after high-intensity interval training (HIIT) and moderate-intensity continuous training (MICT) in rats fed a Western (WD) or normal (ND) diet. Values are expressed as mean \pm SEM. ${ }^{*} P<0.05$ vs. ND+SED. SED: sedentary.

\section{Discussion}

The present study suggests that HIIT and MICT attenuate WD-imparted inflammation in rat myocardium. WD resulted in abdominal (visceral) obesity and that effect was coupled with cardiac TNF- $\alpha$ over-expression (Fig. 4, A). HIIT and MICT counteracted visceral fat mass accumulation, reduced TNF- $\alpha$ expression in the myocardium (Fig. 4, A). Furthermore, HIIT and MICT are both capable of reducing cardiac MPO expression in the ND rats (Fig. 4, B). Our findings attest that HIIT and MICT, although very different in design, appear to be similarly effective in limiting expression of pro-inflammatory cytokines in myocardium of the WD and ND rats. Therefore, this study advocates the anti-inflammatory effects of aerobic training regardless of modality or intensity.

Studies have recognized adipose tissue as an active metabolic-endocrine organ (Greenberg and Obin, 2006). Molecules secreted from adipose tissue have profound effects on immune function (Greenberg and Obin, 2006). Visceral adipose tissue is a major source of inflammation (Alexopoulos et al., 2014), which may contribute to the development of life-style related CVD (Alexopoulos et al., 2014; Singh-Manoux et al., 2017). The relationship between visceral fat and serum TNF- $\alpha$ levels has been supported by clinical research (Schlecht et al., 2016). In this study, WD-induced deposition of fat in the abdominal region was accompanied by cardiac TNF- $\alpha$ over-expression. This observation is in ac- 


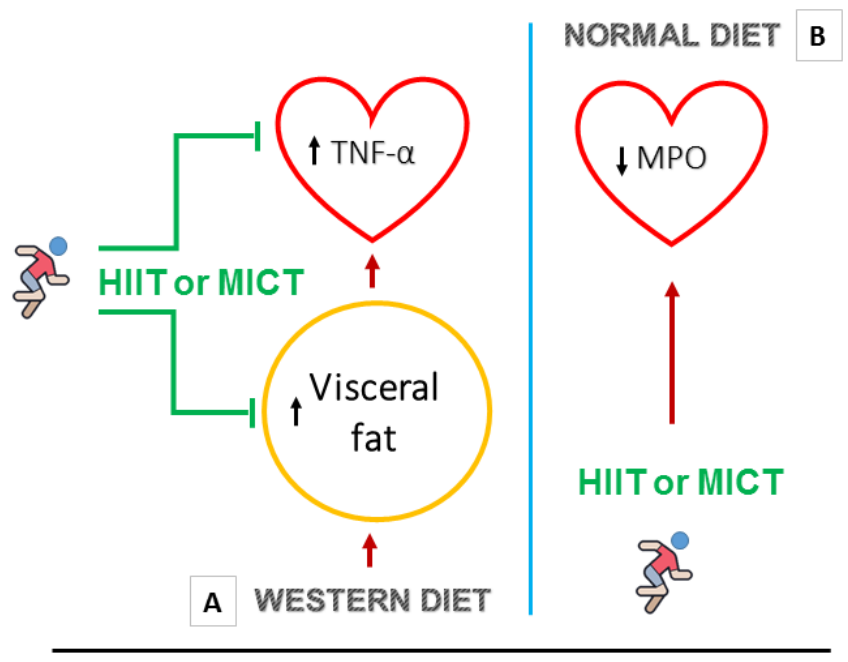

cordance with findings that WD/HFD/obesity increases pro-inflammatory cytokines, both in circulation and heart tissue (Carbone et al., 2017; Fenton et al., 2009; Kesherwani et al., 2015). The mechanisms by which $\mathrm{WD} / \mathrm{HFD} /$ obesity promotes TNF- $\alpha$ expression in the heart still remain unclear. Some likelihood is that visceral adipose tissue releases TNF- $\alpha$ into the circulation (Kesherwani et al., 2015; Schlecht et al., 2016), which in turn, causes activation of cell surface TNF- $\alpha$ receptors in the heart, thereby nuclear factor-kappa beta (NF$к \beta$ ) (Bradham et al., 2002). Activation of NF-к $\beta$ may result in the induction of inflammatory genes, such as those encoding TNF- $\alpha$ and interleukin (IL)- 6 (Lira et al., 2012; Liu et al., 2017; Sarvottam and Yadav, 2014). Beyond that, WD may directly augment the pro-inflammatory mediators, in part, through the binding of saturated fat and sugars to the cardiac pattern recognition receptors, mainly Toll-like receptors (TLR), known as the primary sensors involved in the innate immune response (Li et al., 2014; Wen et al., 2011). It has been suggested that over-expression of the pro-inflammatory cytokine TNF- $\alpha$ by cardiac resident macrophages and cardiomyocytes jeopardizes myocardial contractile function in an autocrine fashion (Meldrum, 1998). In clinical trials, TNF- $\alpha$ antagonists were applied as a potential therapy for heart failure, however, they yielded conflicting results and were not promising (Gupta and Tripathi, 2005). Therefore, exercise training remains still a capstone of the treatment policies for obesity and its related clinical complications.

This study indicated the similar anti-inflammatory effects of HIIT and MICT on rat myocardium, both in WD and ND. Importantly, both modalities of aerobic training
FIGURE 4. A schematic representation of the anti-inflammatory effects of exercise on myocardium in rats fed a Western (WD) or normal diet (ND). WD leads to abdominal obesity, which may be responsible for the enhancement of tumor necrosis factor-alpha $(\mathrm{TNF}-\alpha)$ in heart tissue (A). High-intensity interval training (HIIT) and moderate-intensity continuous training (MICT) are equipotent in obviating WD-induced abdominal obesity and cardiac TNF- $\alpha$ over-expression (A). HIIT and MICT can similarly reduce myeloperoxidase (MPO) expression in the myocardium of the ND rats (B).

not only counteracted cardiac TNF- $\alpha$ over-expression linked to WD but also reduced MPO levels in rats fed a ND. This finding is in accordance with HFD animal studies that aerobic training attenuates pro-inflammatory cytokines in the heart. The anti-inflammatory effects of exercise training may be achieved, in part, by a reduction in visceral adipose tissue (Gleeson et al., 2011). This positive change limits the secretion of adipokines from adipocytes (Gleeson et al., 2011). Further, regular exercise may confer cardioprotection against WD or HFD by increasing IL-10 (Kesherwani et al., 2015) or inhibiting P2X7 purinergic receptors (Chen et al., 2019) in the myocardium. Exercise training releases IL-6 into the circulation by stimulating contracting muscle fibers. The IL-6 myokine has been found to increase and decrease anti-inflammatory IL-10 and pro-inflammatory TNF- $\alpha$, respectively (Benatti and Pedersen, 2015; Petersen and Pedersen, 2005; Steensberg et al., 2003). It is important to note that the ratios of pro- to anti-inflammatory cytokines are essential to fully describe the inflammatory state. This should be considered in future studies.

Our study for the first time demonstrated that WD is not incisive enough to induce MPO protein expression in rat myocardium. It is we speculated that instead of WD an unrelenting condition such as found in myocardial infarction is required for MPO induction in the heart (Ali et al., 2016), although more measurements are essential to leap a conclusion. Future studies should also monitor MPO activity and gene expression, and changes in cardiac neutrophils in the context of WD. In exercise groups, cardiac MPO levels were partially lower in all trained than sedentary rats, which reached statistical 
significance only in ND. At present, it is hard depicting how this occurs. Some likelihood is that HIIT and MICT reduce the source of MPO secretion, namely neutrophils (Ali et al., 2016). Moreover, exercise-induced far-reaching adaptations on cardiac muscle may reduce a requirement for MPO production by unknown mechanisms. MPO inhibition is critical for limiting myocardial dysfunction in heart failure (Ali et al., 2016), but the importance of cardiac MPO modification in intact myocardium by exercise remains to be elucidated.

\section{Conclusion}

Consumption of WD co-existing with sedentary behavior led to abdominal (visceral) obesity which was associated with an increased pro-inflammatory cytokine TNF- $\alpha$ in the myocardium. Exercise intervention, whether HIIT or MICT, ameliorated WD induced visceral obesity and cardiac inflammation. Therefore, this study advocates the cardioprotective effects of aerobic training, regardless of modality or intensity, in those who regularly consume WD.

\section{Acknowledgments}

Our deepest gratitude to Dr. Nazareno Paolocci, Associate Professor at the Johns Hopkins University School of Medicine, for his immense contribution to preparing this manuscript.

\section{Conflict of interest}

The authors declare no conflicts of interest.

\section{References}

Alexopoulos N, Katritsis D, Raggi P. Visceral adipose tissue as a source of inflammation and promoter of atherosclerosis. Atherosclerosis 2014; 233: 104-12. https://doi. org/10.1016/j.atherosclerosis.2013.12.023

Ali M, Pulli B, Courties G, Tricot B, Sebas M, Iwamoto Y, et al. Myeloperoxidase inhibition improves ventricular function and remodeling after experimental myocardial infarction. JACC Basic Transl Sci 2016; 1: 633-43. https://doi. org/10.1016/j.jacbts.2016.09.004

American Physiological Society; World Medical Association General Assembly. Guiding principles for research involving animals and human beings. A $\mathrm{m}$ J Physiol Cell Physiol 2002; 282: 3.

Benatti FB, Pedersen BK. Exercise as an anti-inflammatory therapy for rheumatic diseases-myokine regulation. Nat
Rev Rheumatol 2015; 11: 86-97. https://doi.org/10.1038/ nrrheum.2014.193

Bradham WS, Bozkurt B, Gunasinghe H, Mann D, Spinale FG. Tumor necrosis factor-alpha and myocardial remodeling in progression of heart failure: a current perspective. Cardiovasc Res 2002; 53: 822-30. https://doi.org/10.1016/ S0008-6363(01)00503-X

Butler T, Ashford D, Seymour A-M. Western diet increases cardiac ceramide content in healthy and hypertrophied hearts. Nutr Metab Cardiovasc Dis 2017; 27: 991-8. https:// doi.org/10.1016/j.numecd.2017.08.007

Carbone S, Lee P, Mauro A, Mezzaroma E, Buzzetti R, Van Tassell B, et al. Interleukin-18 mediates cardiac dysfunction induced by western diet independent of obesity and hyperglycemia in the mouse. Nutr Diabetes 2017; 7: e258. https://doi.org/10.1038/nutd.2017.1

Carbone S, Mauro AG, Mezzaroma E, Kraskauskas D, Marchetti C, Buzzetti R, et al. A high-sugar and high-fat diet impairs cardiac systolic and diastolic function in mice. Int J Cardiol 2015; 198: 66-9. https://doi.org/10.1016/j.ijcard.2015.06.136

Catta-Preta M, Martins MA, Brunini TMC, Mendes-Ribeiro AC, Mandarim-de-Lacerda CA, Aguila MB. Modulation of cytokines, resistin, and distribution of adipose tissue in C57BL/6 mice by different high-fat diets. Nutrition 2012; 28: 212-9. https://doi.org/10.1016/j.nut.2011.05.011

Chavali V, Tyagi SC, Mishra PK. Differential expression of dicer, miRNAs, and inflammatory markers in diabetic Ins2+/- Akita hearts. Cell Biochem Biophys 2014; 68: 2535. https://doi.org/10.1007/s12013-013-9679-4

Chen X, Li H, Wang K, Liang X, Wang W, Hu X, et al. Aerobic exercise ameliorates myocardial inflammation, fibrosis and apoptosis in high-fat-diet rats by inhibiting $\mathrm{P} 2 \mathrm{X} 7$ purinergic receptors. Front Physiol 2019; 10: 1286. https://doi. org/10.3389/fphys.2019.01286

Chen Y, Feng B, Yuan Y, Hu J, Zhao W, Jiang H, et al. Aloe emodin reduces cardiac inflammation induced by a high-fat diet through the TLR4 signaling pathway. Mediators Inflamm 2020; 2020. https://doi.org/10.1155/2020/6318520

Costa RR, Villela NR, Maria das Graças CS, Boa BC, Cyrino FZ, Silva SV, et al. High fat diet induces central obesity, insulin resistance and microvascular dysfunction in hamsters. Microvasc Res 2011; 82: 416-22. https://doi.org/10.1016/j. mvr.2011.08.007

Delwing-de Lima D, Ulbricht ASSF, Werlang-Coelho C, Delwing-Dal Magro D, Joaquim VHA, Salamaia EM, et al. Effects of two aerobic exercise training protocols on parame- 
ters of oxidative stress in the blood and liver of obese rats. J Physiol Sci 2018; 68: 699-706. https://doi.org/10.1007/ s12576-017-0584-2

Ellison GM, Waring CD, Vicinanza C, Torella D. Physiological cardiac remodelling in response to endurance exercise training: cellular and molecular mechanisms. Heart 2012; 98: 5-10. https://doi.org/10.1136/heartjnl-2011-300639

Fenton JI, Nunez N, Yakar S, Perkins S, Hord N, Hursting $\mathrm{S}$. Diet-nduced adiposity alters the serum profile of inflammation in C57BL/6N mice as measured by antibody array. Diabetes Obes Metab 2009; 11: 343-54. https://doi. org/10.1111/j.1463-1326.2008.00974.x

Fontana L, Eagon JC, Trujillo ME, Scherer PE, Klein S. Visceral fat adipokine secretion is associated with systemic inflammation in obese humans. Diabetes 2007; 56: 1010-3. https://doi.org/10.2337/db06-1656

Gleeson M, Bishop NC, Stensel DJ, Lindley MR, Mastana SS, Nimmo MA. The anti-inflammatory effects of exercise: mechanisms and implications for the prevention and treatment of disease. Nat Rev Immunol 2011; 11: 607-15. https://doi.org/10.1038/nri3041

Greenberg AS, Obin MS. Obesity and the role of adipose tissue in inflammation and metabolism. The Am J Clin Nutr 2006; 83: 461S-5S. https://doi.org/10.1093/ajen/83.2.461S

Gupta S, Tripathi CD. Practitioners'section-current status of tnf blocking therapy in heart failure. Indian J Med Sci 2005; 50:363-366. https://doi.org/10.4103/00195359.16655

Hafstad AD, Lund J, Hadler-Olsen E, Höper AC, Larsen TS, Aasum E. High-and moderate-intensity training normalizes ventricular function and mechanoenergetics in mice with diet-induced obesity. Diabetes 2013; 62: 2287-94. https:// doi.org/10.2337/db12-1580

Kalász J, Pásztor ET, Fagyas M, Balogh Á, Tóth A, Csató $\mathrm{V}$, et al. Myeloperoxidase impairs the contractile function in isolated human cardiomyocytes. Free Radic Biol Med 2015; 84: 116-27. https://doi.org/10.1016/j.freeradbiomed.2015.02.036

Kesherwani V, Chavali V, Hackfort BT, Tyagi SC, Mishra PK. Exercise ameliorates high fat diet induced cardiac dysfunction by increasing interleukin 10. Front Physiol 2015; 6: 124. https://doi.org/10.3389/fphys.2015.00124

Koeth RA, Haselden V, Tang WW. Myeloperoxidase in cardiovascular disease. Adv Clin Chem 2013; 62: 1-32. https:// doi.org/10.1016/B978-0-12-800096-0.00001-9

Li X, Du N, Zhang Q, Li J, Chen X, Liu X, et al. MicroRNA-30d regulates cardiomyocyte pyroptosis by direct- ly targeting foxo3a in diabetic cardiomyopathy. Cell Death Dis 2014; 5: e1479-e. https://doi.org/10.1038/cddis. 2014.430

Lira FS, Rosa JC, Pimentel GD, Seelaender M, Damaso AR, Oyama LM, et al. Both adiponectin and interleukin-10 inhibit LPS-induced activation of the NF- $\kappa B$ pathway in 3T3-L1 adipocytes. Cytokine 2012; 57: 98-106. https://doi. org/10.1016/j.cyto.2011.10.001

Liu T, Zhang L, Joo D, Sun S-C. NF-кB signaling in inflammation. Signal Transduct Target Ther 2017; 2: 1-9. https:// doi.org/10.1038/sigtrans.2017.23

Lumeng CN, Saltiel AR. Inflammatory links between obesity and metabolic disease. J Clin Invest 2011; 121: 2111-7. https://doi.org/10.1172/JCI57132

Machado MV, Vieira AB, da Conceição FG, Nascimento AR, da Nóbrega ACL, Tibirica E. Exercise training dose differentially alters muscle and heart capillary density and metabolic functions in an obese rat with metabolic syndrome. Exp Physiol 2017; 102: 1716-28. https://doi.org/10.1113/ EP086416

McGavock JM, Anderson TJ, Lewanczuk RZ. Sedentary lifestyle and antecedents of cardiovascular disease in young adults. Am J Hypertens 2006; 19: 701-7. https://doi. org/10.1016/j.amjhyper.2006.01.013

Meldrum DR. Tumor necrosis factor in the heart. Am J Physiol 1998; 274: 577-95. https://doi.org/10.1152/ajpregu.1998.274.3.R577

Mishra PK, Awe O, Metreveli N, Qipshidze N, Joshua IG, Tyagi SC. Exercise mitigates homocysteine- $\beta 2$-adrenergic receptor interactions to ameliorate contractile dysfunction in diabetes. Int J Physiol Pathophysiol Pharmacol 2011; 3: 97.

Moreno-Fernández S, Garcés-Rimón M, Vera G, Astier J, Landrier JF, Miguel M. High fat/high glucose diet induces metabolic syndrome in an experimental rat model. Nutrients 2018; 10: 1502. https://doi.org/10.3390/nu10101502

Mostarda C, Moraes-Silva IC, Salemi VMC, Machi JF, Rodrigues B, De Angelis K, et al. Exercise training prevents diastolic dysfunction induced by metabolic syndrome in rats. Clinics 2012; 67: 815-20. https://doi.org/10.6061/clinics/2012(07)18

Mozaffarian D, Benjamin EJ, Go AS, Arnett DK, Blaha MJ, Cushman M, et al. Executive summary: heart disease and stroke statistics-2016 update: a report from the American Heart Association. Circulation 2016; 133: 447-54. https:// doi.org/10.1161/CIR.0000000000000366

Nystoriak MA, Bhatnagar A. Cardiovascular effects and 
benefits of exercise. Front Cardiovasc Med 2018; 5: 135. https://doi.org/10.3389/fcvm.2018.00135

Oikonomou E, Psaltopoulou T, Georgiopoulos G, Siasos G, Kokkou E, Antonopoulos A, et al. Western dietary pattern is associated with severe coronary artery disease. Angiology 2018; 69: 339-46. https://doi. org/10.1177/0003319717721603

Panagiotakos D, Pitsavos C, Chrysohoou C, Palliou K, Lentzas I, Skoumas I, et al. Dietary patterns and 5-year incidence of cardiovascular disease: a multivariate analysis of the ATTICA study. Nutr Metab Cardiovasc Dis 2009; 19: 253-63. https://doi.org/10.1016/j.numecd.2008.06.005

Pedersen BK. Anti-inflammatory effects of exercise: role in diabetes and cardiovascular disease. Eur J Clin Invest 2017; 47: 600-11. https://doi.org/10.1111/eci.12781

Petersen AMW, Pedersen BK. The anti-inflammatory effect of exercise. J Appl Physiol 2005; 98: 1154-62. https://doi. org/10.1152/japplphysiol.00164.2004

Rariden BS, Boltz AJ, Brawner CA, Pinkstaff SO, Richardson MR, Johnson TM, et al. Sedentary time and cumulative risk of preserved and reduced ejection fraction heart failure: from the multi-ethnic study of atherosclerosis. J Card Fail 2019; 25: 418-24. https://doi.org/10.1016/j.cardfail.2019.03.017

Santana ABC, Souza Oliveira TCd, Bianconi BL, Barauna VG, Santos EWCO, Alves TP, et al. Effect of highfat diet upon inflammatory markers and aortic stiffening in mice. Biomed Res Int 2014; 2014. https://doi. org/10.1155/2014/914102

Sarvottam K, Yadav RK. Obesity-related inflammation \& cardiovascular disease: efficacy of a yoga-based lifestyle intervention. Indian J Med Res 2014; 139: 822. Schlecht I, Fischer B, Behrens G, Leitzmann MF. Relations of visceral and abdominal subcutaneous adipose tissue, body mass index, and waist circumference to serum concentrations of parameters of chronic inflammation. Obes Facts 2016; 9: 144-57. https://doi.org/10.1159/000443691

Shephard RJ, Balady GJ. Exercise as cardiovascular therapy. Circulation 1999; 99: 963-72. https://doi.org/10.1161/01. CIR.99.7.963

Singh-Manoux A, Shipley MJ, Bell JA, Canonico M, Elbaz A, Kivimäki M. Association between inflammatory biomarkers and all-cause, cardiovascular and cancer-related mortality. CMAJ 2017; 189: E384-90. https://doi.org/10.1503/ cmaj. 160313

Steensberg A, Fischer CP, Keller C, Møller K, Pedersen BK. IL-6 enhances plasma IL-1ra, IL-10, and cortisol in humans. Am J Physiol Endocrinol Metab 2003; 285: E433-7. https://doi.org/10.1152/ajpendo.00074.2003

Verboven M, Cuypers A, Deluyker D, Lambrichts I, Eijnde BO, Hansen D, et al. High intensity training improves cardiac function in healthy rats. Sci Rep 2019; 9: 1-8. https:// doi.org/10.1038/s41598-019-42023-1

Wen H, Gris D, Lei Y, Jha S, Zhang L, Huang MT-H, et al. Fatty acid-induced NLRP3-ASC inflammasome activation interferes with insulin signaling. Nat Immunol 2011; 12: 408-15. https://doi.org/10.1038/ni.2022

Xi Y, Gong D-W, Tian Z. FSTL1 as a potential mediator of exercise-induced cardioprotection in post-myocardial infarction rats. Sci Rep 2016; 6: 1-11. https://doi.org/10.1038/ srep32424 